\title{
THE EFFECT OF DIFFERENT SEALER VOLUME ON BOND STRENGTH OF TOTAL FILLS BC OBTURATING SYSTEM
}

\author{
Nancy Magdy M.El-said", Salma Hassan El-ashry ${ }^{* *}$ and Amira Galal Ismail ${ }^{* * *}$
}

\begin{abstract}
The bond strength of Total fill BC obturation system using single cone size 40 and size 50 at different time intervals; 1days and 7 days was evaluated in this vitro study. Twenty four single rooted single canal extracted human teeth were used in this study. All the teeth were instrumented with a crown down technique by Endo Star taper 4\% to achieve size \#50 apical preparation .irrigation was done using $10 \mathrm{ml} 2.25 \% \mathrm{Na}(\mathrm{ocl})$, then $10 \mathrm{ml}$ of $17 \%$ EDTA and final rinse with $10 \mathrm{ml}$ sterile normal saline. Single cone technique was used for obturating all the groups as the manufacturer's instructions. The samples were classified into two main groups according to the size of the obturating material ( $\mathrm{n}=12)$; group "I" canals were obturated with Totalfill points size 50 and group "II" (canals were obturated with Totalfill points size 40. Each group was subdivided into 2 subgroups according to the time of the test $(\mathrm{n}=6)$; subgroup " 1 ' examined after 1 day and Subgroup "2" examined after 7 days. Each subgroup is tested using universal testing machine to evaluate the push out Bond strength. After 1 day; there was no statistically significant difference between the two groups either obturated with size 50 or obturated with size 40 , However After 7 days regardless of root level, the Group obturated with size 50 showed statistically significantly higher median push-out bond strength than Group obturated with size 40 it was concluded that The bond strength is higher with the large size of gutta percha core than the volume of sealer.
\end{abstract}

\section{INTRODUCTION}

Obturation of root canal complex is the most essential step in successful root canal therapy. It is described as "the three dimensional closure of the entire root canal system adapted to the cement- dentinal junction as close as possible". For an appropriate and acceptable root canal fluid tight seal; Small volume of sealers which are biologically compatible are used along with gutta percha core at apical, lateral and coronal sections of root canal system. ${ }^{(1)}$

\footnotetext{
* Endodontics, Faculty of Dentistry, Misr International University

** Faculty of Oral And Dental Medicine, Department Of Endodontics, Ain Shams Univerisity

*** National Research Center, Department of Endodntics, Dentistry.
} 
New obturating material has been developed in Endodontics to enhance the sealing of the root canal system. Bio-ceramics were introduced; bioceramics are calcium silicate based material which exhibit excellent biocompatibility properties due to their similarity with biological hydroxyapatite, bioceramics also have the ability to achieve excellent hermetic seal, form a chemical bond with the tooth structure and have good radio-opacity antibacterial properties as a result of precipitation in situ after setting.

Total fill as one of the advances in bioceramics; it is bioceramic impregnated gutta percha with a premixed bioceramic sealer. It was claimed that the calcium phosphate-based system made for a more consistent and tighter adaptation to the dentinal walls as compared to gutta-percha. Total Fill BC sealer has higher qualities and handling abilities that prevents its shrinkage during setting and hardens in the presence of moisture. The wetting ability of the sealer is due to the small particle size, hydro-philicity and low contact angle; these properties enable the sealer to easily distribute in the dentinal tubules. It also shows a significant expansion of 0.20 percent. These characteristics create a gapless chemical bond between the sealer and dentinal walls. ${ }^{(2,3)}$

It is worth to study and evaluate the bond strength, and effect of different volume of sealers on these properties.

\section{MATERIALS AND METHODS}

All the 24 teeth were cleaned from any remnants of hard calculus using ultra-sonic scaler and were immersed in $5.25 \%$ sodium hypochlorite for 15 minutes, after that, teeth were preserved in $0.9 \%$ sterile normal saline at room temperature.

The crown is removed to Standardized the root length to be $15 \mathrm{~mm}$.Apical patency was insured by $\mathrm{k}$-file size 10 .Working length was established with size $15 \mathrm{k}$ file into the canal until observed at the apical foramen. Then the file was withdrawn. $1 \mathrm{~mm}$ was subtracted from the measured length. The canals were shaped following the crown down technique, by Endo Star taper 4\% following the manufacturer's instructions fig (3); with sequence iso3, iso4, iso5, iso35, iso40, iso45, iso. All the canals instrumented till reached size ISO 50 taper 4\%, recapitulation was done between each rotary file by size $15 \mathrm{k}$ - file to avoid accumulation of debris combined with corpus irrigation .Irrigation was done using $5 \mathrm{ml}$ of $2.5 \%$ sodium hypochlorite between every change of files (using side perforating needles).

After instrumentation, the teeth received a final rinse with $10 \mathrm{ml}$ of $17 \%$ aqueous solution ethylene diamine terta-acetic acid chelating agent for removal of smear layer and $10 \mathrm{ml}$ of $2.25 \%$ sodium hypochlorite followed by final rinse with $10 \mathrm{ml}$ of sterile normal saline and dried with paper-points.

Single cone technique was used for all the groups as the manufacturer's instructions. Group I were trial fitted to achieve tug-back and a radiograph was taken to confirm the position of the Totalfill points Size 50.Fasten the BC tip firmly to the syringe hub with a clockwise twist. BC tips are adjustable and can be twisted to allow the root canal to be reached by pushing the syringe tip into the canal no deeper than the coronal one third. Kindly and easily dispense 2 Totalfill BC sealer guide marks into the root canal by compressing the syringe plugger Using a \# 15 hand-file, coat the canal walls lightly with the current sealer in the canal, then cover the master gutta percha cone with a thin layer of sealer and insert it very slowly into the channel. The master gutta percha brings enough sealant to the apex. The excess Totalfill points were trimmed to the level of the canal orifice using hot plugger.

Group II were obturated with the same sequence as group ' $A$ ' except for the size; Totalfill points size 40 and 3reference markings of Totalfill BC sealer.

Radiographs were taken for all teeth to verify the root canal filling. 
Groups to be evaluated after 7 days were placed in the incubator to mimic the oral environmental conditions $37^{\circ} \mathrm{C} \pm 0.1^{\circ} \mathrm{c}$.

For testing the push out pond strength test: Each tooth was mounted in a specially designed cylindrical Teflon molds made of polymethylmethacrylate (PMMA) resin blocks of $4 \mathrm{~cm}$ length $\mathrm{x}$ $2 \mathrm{~cm}$ width $\times 3 \mathrm{~cm}$ height so the crowns were upside down inside the mold. The molds were sectioned by precision saw using water cooled diamond disc of 4 inch diameter $\mathrm{x} 0.5 \mathrm{~mm}$ thickness with blade speed $2500 \mathrm{rpm}$ under continuous water spray .The samples were sectioned perpendicular to the long axis of each root to examine the cross section of the root .Each root was cut to get three sections; apical, middle and coronal, $2 \mathrm{~mm}$ thickness from each section. They were color coded red, blue and black for coronal, middle and apical respectively. The discs present were subjected to compressive load via the universal testing machine with a crosshead speed of $1 \mathrm{~mm}$ per minute using a $0.8 \mathrm{~mm}$ diameter plugger for the coronal one third, $0.6 \mathrm{~mm}$ for the middle one third and $0.4 \mathrm{~mm}$ for the apical one third. The plugger only contacted the obturating material. The push out force was applied in an apico-coronal direction until bond failure occurs and the filling material is extruded from the disc. Results were recorded and manipulated by data analysis software.

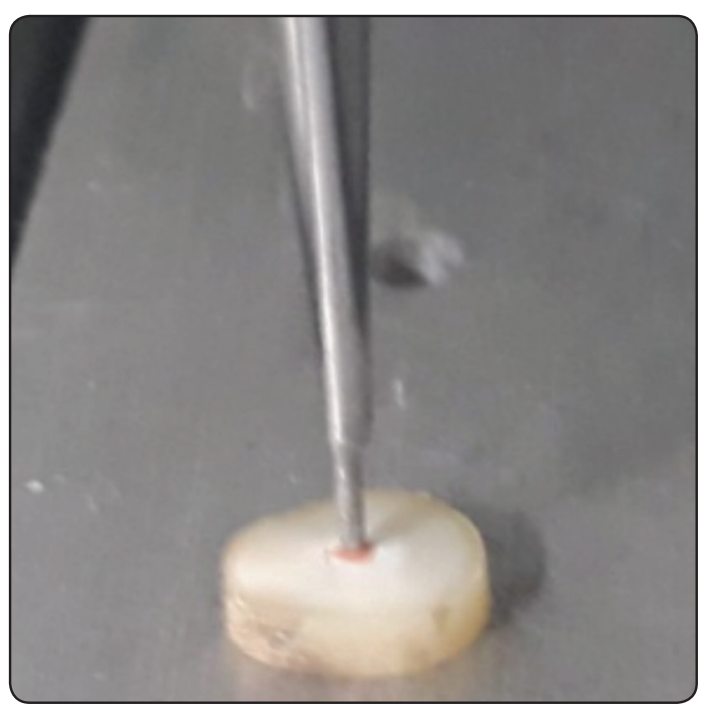

Fig. (1)
The push out bond strength was calculated in mega-pascals according to the following formula:

Pushout bond strength $(\mathrm{MPa})=$ MAXIMUM LOAD (N)

Adhesion area of root canal filling (mm2)

The adhesion area was calculated using the following formula: $\pi(\mathrm{R}+\mathrm{r})$ [h2 $+(\mathrm{R}-\mathrm{r})$ 2]0.5In which $\pi=3.14$, $R$ is the coronal radius, $r$ is the apical radius and $\mathrm{h}$ is the slice thickness. ${ }^{(4)}$

Data were presented as mean, standard deviation (SD), median, and range. All statistics revealed a non-normal distribution (non-parametric one) The Mann-Whitney U method was used to evaluate the two categories as well as the time intervals between them. The test carried out by Friedman was used to compare root levels within each group. Dunn's test used for pair-wise analyses. The level of importance was set at 0.05 P. Statistical research was carried out for Windows with IBM SPSS Statistics, version 23.0. Armonk, New York: IBM Corp.

\section{RESULT}

Comparing group I and group II After 1 day; there was no statistically significant difference between the two groups at the apical, middle as well as coronal root levels $(\mathrm{P}$-value $=0.337$, Effect size $=0.577),(\mathrm{P}$-value $=0.262$, Effect size $=0.684)$ and $(\mathrm{P}$-value $=0.631$, Effect size $=0.280)$, respectively. Similarly for the total push-out bond strength regardless of root level, there was no statistically significant difference between the two groups $(\mathrm{P}$-value $=0.749$, Effect size $=0.186)$.

Comparing the two groups After 7 days; Group I showed statistically significantly higher median push-out bond strength than Group II at the apical and middle root levels (P-value $=0.016$, Effect size $=1.925)$ and $(\mathrm{P}$-value $=0.004$, Effect size $=3.000)$, respectively. While at coronal root level, there was no statistically significant difference between the two groups $(\mathrm{P}$-value $=0.078$, Effect size $=$ 1.181). As regards the total push-out bond strength 
regardless of root level, Group I showed statistically significantly higher median push-out bond strength than Group II (P-value $=0.004$, Effect size $=3.000)$.

When comparing the time intervals for the total push-out bond strength between 1 day and 7 days in group I regardless of root level, there was no statistically significant difference between the two time periods $(P$-value $=0.337$, Effect size $=0.577)$.

When comparing the time intervals for the total push-out bond strength between 1 day and 7 days in group II regardless of root level, there was no statistically significant difference between the two time periods $(P$-value $=1.000$, Effect size $=0.000)$.

When comparing the root levels for the total push-out bond strength in group I regardless of root level, there was no statistically significant difference between the root levels either after 1 day or after 7 days $(P$-value $=0.069$, Effect size $=0.444)$.and $(P$-value $=0.311$, Effect size $=0.194)$.respectively .

When comparing the root levels for the total push-out bond strength in group II regardless of root level, there was no statistically significant difference between the root levels either after 1 day or after 7 days $(P$-value $=0.311$, Effect size $=0.194)$.and $(P$-value $=0.607$, Effect size $=0.083)$.respectivel.

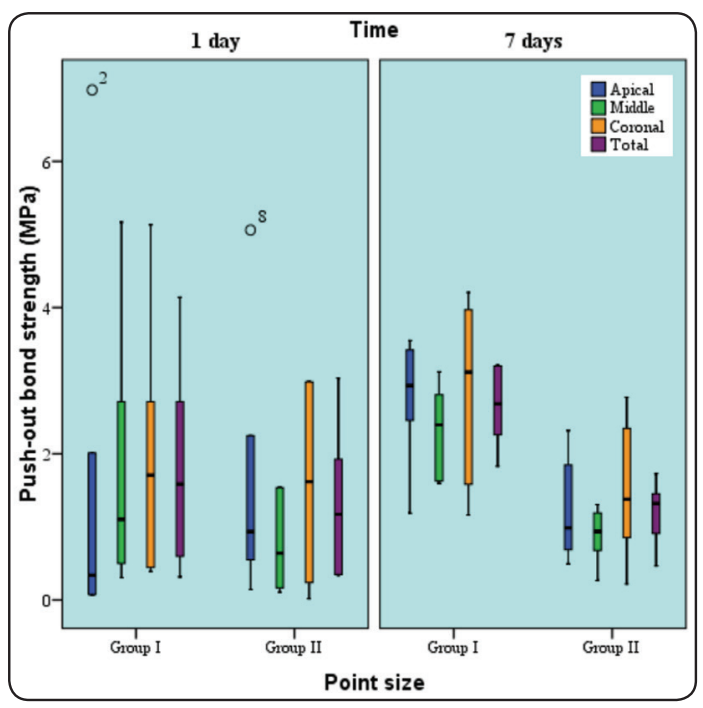

Fig. (2)
Box plot representing median and range values for push-out bond strength of the two groups (Point sizes) (Circles represent outliers)

\section{DISCUSSION}

A wide range of sealers have been used in endodontics, newly introduced is total Fill BC sealer. The endodontic bioceramic sealer is formed of silicate calcium and phosphate calcium groups. Total Fill BC sealer has the same structure as Endosequence $\mathrm{BC}$ sealer and has both superior physical and excellent biocompatibility characteristics. Total Fill BC sealer is created of superior qualities and handling capabilities as an innovative novel root canal sealer that does not shrink during setting and hardens in the presence of moisture. In comparison to traditional filling cements, the TotalFill BC sealer setting reaction is caused by the moisture present in the dentinal tubules. Total Fill BC Sealer absorbs the moisture to form hydroxyapatite to achieve maximum chemical adhesion between the cement and the dentin. ${ }^{(5)}$

This sealer's small particle size, hydrophilicity, and low contact angle allow it to quickly disperse over the canal's dentinal walls and get inside and fill the lateral micro-channels. It also indicates a substantial 0.20 percent growth. These characteristics create a gapless chemical bond between the sealer and the dentinal walls. About two hours later the setting reaction of Total Fill BC sealer triggered by moisture and the final set was completed with the calcium silicate component of the substance creating a calcium silicate hydrate gel and calcium hydroxide. Then the calcium hydroxide interacted with the dentine phosphate ions to form hydroxyapatite and water. The water generated proceeded to interact with the silicate of calcium to precipitate extra gel-like silicate hydrate ${ }^{(6)}$

In the current study, we compared the single cone technique with bio-ceramic calcium silicate sealer obturation technique by using two sizes (50 and 40). 
The null hypothesis is that there is no significant difference between the two sizes in bond strength and adaptation as regards to the amount of sealer.

Extracted single human teeth were utilized in the current study to simulate the clinical condition.

All the canals preparations reached size ISO 50 taper $4 \%$, recapitulation was done between each rotary file by size $15 \mathrm{k}$-file to avoid accumulation of debris combined with copious irrigation. Irrigation was done using $5 \mathrm{ml}$ of $2.5 \%$ sodium hypochlorite between each change of files (using side perforating needles to mimic the clinical condition). ${ }^{(7)}$

After instrumentation, the teeth received a final flush with $10 \mathrm{ml}$ of $17 \%$ aqueous solution ethylene-diamine-terta-acetic acid chelating agent (EDTA) for removal of smear layer and $10 \mathrm{ml}$ of $2.25 \%$ sodium hypochlorite followed by final rinse with $10 \mathrm{ml}$ of sterile normal saline and dried with paper-points. ${ }^{(8)}$

In this analysis, EDTA (17 percent) was used as a final irrigant as it was seen to improve the wetting potential of dentinal walls, thereby providing an atmosphere appropriate for adhesion of hydrophilic materials such as TotalFill BC. Hence, Totalfill BC sealer's wetting and flow-ability allowed penetration into the open dentinal tubules and produced successful micro-retention. The reduced wetting ability of the dentin surface therefore prevented the adherence to any hydrophilic materials. Bioceramic sealants are hydrophilic by definition, meaning that their environment and adhesion are a function of the root canal moisture. ${ }^{(9)}$

Single cone technique was used in this research, as it is gaining popularity in clinical practice since it is an easy and fast technique; in 2007 Bindslve et al demonstrated in his study that there is no significant difference in the treatment findings after 6 to 18 months between single cone and lateral compaction. ${ }^{(10)}$

In 2013, Collares et al ${ }^{(17)}$ found that the singlecone technique had higher dislodging resistance when compared to the resistance demonstrated techniques which used more than one cone. On the other hand, because of its cohesive strength, sealers with high mechanical properties could hinder the result values. ${ }^{(11)}$

Related criteria for measuring bond strength have been published. For direct comparison of the results, shear strength, micro-tensile and even pullout or push-out tests were described as reproducible and effective. Push-out test enables precise standardization of the specimen's .However, it is clear that research models are unlikely to replicate the same clinical conditions, primarily because root dentin is not standardized and the composition of the canal walls prepared for the endodontic procedure is substantially different. In several studies the thickness of dentin used for push-out tests has also been variable ${ }^{(12,13)}$. Root slices were designed, from $0.6 \mathrm{~mm}$ to $7.0 \mathrm{~mm}$. In order to produce a greater number of samples and even strong bond strength values are required, thin slices, around $1.0 \mathrm{~mm}$ thick, appear preferred. As stated by Gesi, et al. ${ }^{(14)}$ Otherwise there is a chance of sealer detachment while slicing.

In this study the push-out test is used as it is easy to reproduce, to interpret, less technique sensitive and common in laboratory studies. ${ }^{(15)}$ The best available data for determining a root canal sealer comes from experimental research, but the findings and methods show considerable variation. Since standardization of such studies is compulsory, it is of paramount importance to investigate the influence of several variables on the resistance to dislodgement and the magnitude of the effect of each variable.

The present research showed that after one day evaluation the total median of bond strength of group I is 1.59 while that of group II is 1.17 which means that group I has more bond strength and this may be due to the larger taper of the master cone present in group I and also due to the prolonged 
setting time of the sealer ( not fully set therefore it's not harden yet), however there was no statistically significant difference between the two groups at the apical, middle as well as coronal root levels.

The total push-out bond strength regardless of root level, there was no statistically significant difference between the two groups. Although the median push out bond strength apically in group I (0.34) is less than the median push out bond strength apically in group II (0.94). Group II has more push out bond strength only apically because that gutta percha fit and more amount of sealer as the BC Sealer expands slightly $(<0.1 \%)$, which may provide superiority for the bond strength when used with the Single Cone technique.

While after 7 days, the total median of bond strength of group I is 2.68 while that of group II is 1.32 which means that group I has more bond strength and this is also may be due to the larger taper of the master cone present in group I .Group I showed statistically significantly higher median push-out bond strength than Group II at the apical and middle root levels. While at coronal root level, there was no statistically significant difference between the two groups. As regards the total pushout bond strength regardless of root level, Group I showed statistically significantly higher median push-out bond strength than Group II. There is an instant adhesion because the bioceramic impregnated gutta percha chemically bond to form hydroxyl apatite crystals and therefore increase the bond strength value. The bioceramic sealer and bond to the dentine wall formed Monoblock.

These results were fully in agreement with the DeLong et al study ${ }^{(6)}$, which determined the impact of obturation technique on calcium silicate sealers' push-out bond strength. They concluded that when used with single cone technique, bio-ceramic and MTA Plus sealers displayed desirable bond strengths
Under the limitations of this study,; it was concluded that The bond strength is higher with the large size of gutta percha core than the volume of sealer and the use of bioceramic Totalfill point and Totalfill BC sealer in a single cone obturation technique has high push out bond strength especially after full setting of the sealer. Bioceramics is not yet evidence based obturating material since it needs more researches considering the durability on the long run, with more clinical trials. And more researches for its retreatment; since the gutta percha cones are impregnated with nano- bioceramic particles and instead of being coated

\section{REFERENCES}

1. American Association of Endodontics: Glossary, contemporary terminology for endodontics, Ed 6, Chicago, The Association, 1994.

2. Han L, Okiji T. Bioactivity evaluation of three calcium silicate-based endodontic materials. Int Endod J 2013; $46: 808-14$

3. Ersahan S, Aydin C. Dislocation resistance of iRoot SP, a calcium silicate based sealer, from radicular dentine. J Endod 2010; 36:2000-2.

4. Bayram H, Brayam E, Kanber M, Celikten B, Saklar F. Effect of different chelating solutions on the push- out bond strength of various root canal sealers. Biomed Res 2017; (Special issue): S401-6

5. Huffman, B.P.; Mai, S.; Pinna, L.; Weller, R.N.; Primus, C.M.; Gutmann, J.L.; Pashley, D.H.; Tay, F.R. Dislocation resistance of prorootendo sealer, a calcium silicatebased root canal sealer, from radicular dentine. Int. Endod. J. 2009, 42, 34-46.

6. Pane, E.S.; Palamara, J.E.A.; Messer, H.H. Critical evaluation of the push-out test for root canal fillingmaterials. J. Endod. 2013, 39, 669-673.

7. Arslan, H.; Abbas, A.; Karatas, E. Influence of ultrasonic and sonic activation of epoxy-amine resin-basedsealer on penetration of sealer into lateral canals. Clin. Oral Investig. 2016, 20, 2161-2164.

8. Zang W, Li Z, Peng B. Assessment of new root canal sealers apical sealing ability. Oral Surg Oral Med Oral Pathol Oral RadiolEndod 2009; 107(6): 79-82. 
9. Sirisha K, Rambabu T, Shankar Y and Ravikumar P :Validity of bond strength tests:A critical review: part I. J Conserv Dent 2014:17(4) :305-3011.

10. Carvalho NK, Prado MC, Senna PM, Neves AA, Souza EM, Fidel SR, Sassone LM, Silva EJ. Do smear-layer removal agents affect the push-out bond strength of calcium-silicate based endodontic sealers? IntEndod J 2017; 50: 612-9.

11. Camilleri J. Sealers and warm gutta-percha obturation techniques. Journal of Endodontics. 2015; 41, 72-8.

12. Dave Pet al. Analysis of Root Canal Obturation Techniques with Gutta Percha. Journal of Advanced Medical and Dental Sciences Research IVol. 7IIssue 2| February 2019
13. Becce C, Pameijer CH. SEM study of a new endodontic root canal sealer. J Dent Res 2001;79

14. Barbizam J TM, Tanomaru-Filho M, Teixeira E, Teixeira F. Bond strength of different endodontic sealers to dentine: Push-out test. J Appl Oral Sci. 2011;19(6):644-7.

15. Gandolfi MG, Siboni F, Prati C. Properties of a novel polysiloxaneguttaperchacalcium silicate-bioglass-containing root canal sealer. Dent Mater. 2016;32(5):e113-e126.

16. Pane ES, Palamara JE, Messer HH. Critical evaluation of the push-out test for root canal filling materials. Jour of Endod 2013; 39, 669-73.

17. Camille to J . Sealers and warm Gutta percha obturation techniques. Journal of endodontics. 2015;41,72-8. 